\title{
Prävention von oralen Erkrankungen
}

\section{Matthias Folwaczny, Reinhard Hickel}

Poliklinik für Zahnerhaltung und Parodontologie, Klinikum der Ludwig-Maximilians Universität München

\section{Prevention of oral diseases}

The different types of hard- and soft tissues that are involved in the formation of the oral cavity are subject of numerous diseases. Albeit dental caries and periodontitis comprise the most common oral disease entities, also several premalignant and malignant conditions can occur within the oral cavity. There exists are wide armamentarium for the prevention of both, dental caries and periodontitis. Preventive strategies are even more important regarding oral malignancies since specifically these entities commonly provide only poor prognosis if not treated in early stages. The present contribution aims to present the pathogenic background of the most common oral diseases and the most effective methods for their prevention.

eingereicht: 30.3.2004 akzeptiert: 8.7.2004

Epidemiologie oraler Erkrankungen

Die als funktionelle Einheit am Aufbau der Mundhöhle beteiligten Gewebekompartimente können von einerVielzahl unterschiedlicher Erkrankungsbilder erfasst werden. Neben der klassischen Zahnkaries und der marginalen Parodontitis sind vor allem die Mundschleimhauterkrankungen einschließlich der Malignome der Mundhöhle zu nennen.

Die außerordentlich große Bedeutung sowohl der dentalen Karies als auch der Parodontitis verdeutlichen epidemiologische Daten, wonach die Prävalenz der dentalen Karies trotz insgesamt abnehmender Tendenz (ausschließlich Fissurenkaries) bereits im Kindesalter bei fast $40 \%$ liegt (Pistorius et al.:

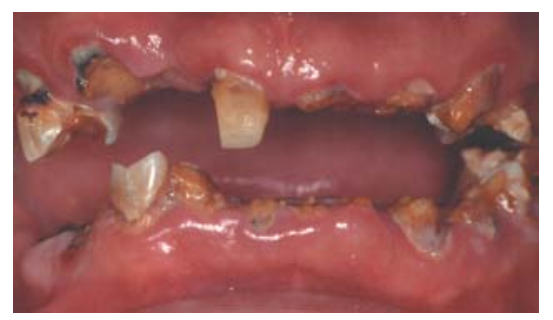

Abb. 1 Fortgeschrittene dentale Karies mit ausgedehnten primärkariösen Defekten (Quelle: M. Folwaczny). lierung einer pathogenen mikrobiellen Besiedelung der freien Zahnoberfläche bzw. des dento-gingivalen Kompartiments, also des Zahnfleischsulcus, angesehen werden (Ximenez-Fyvie et al.: J Clin Periodontol 2000; 27: 722). Bei beiden Erkrankungen formiert sich die pathogene Bakterienflora in Symbiose mit komensalischen Bakterien der Mundhöhle in Form eines Biofilms. Die typischen Erkrankungssymptome resultieren nicht aus der Infektion durch Bakterien einer singulären Spezies. Vielmehr ermöglichterst das Zusammenspiel zahlreicher verschiedener, bis zu 300 unterschiedlicher Bakterienspezies, die Etablierung der eigentlichen krankheitsauslösenden Bakterien (Socransky et al.: J Clin Periodontol 1998; 25: 134).

Die dentale Karies wird im wesentlichen durch Bakterien der Spezies Streptococcus mutans sowie durch Lactobacillus spp. induziert. Der herausragende Virulenzmechanismus dieser Bakterien ist die Produktion von Säuren, die letztendlich zur Demineralisation und Kavitation der Zahnoberfläche führen. Zusätzlich ermöglicht die Produktion von schwer löslichen Polysacchariden als Teil der extrazellulären Biofilmmatrix durch Str. mutans den Bakterienzellen die feste Anheftung an die Zahnoberfläche. Im Zusammenhang mit der Parodontitis wird derzeit insgesamt sieben Bakterienspezies ein hohes pathogenes Potenzial zugeordnet: Actinobacillus actinomycetemcomitans, Porphyromonas gingivalis, Tanerella forsythensis, Prevotella intermedia, Treponema denticola und Eubacterium nodatum (Socransky et al.: Ann Periodontol 1997; 2: 3). Die parodontopathogenen Bakterien induzieren unter Vermittlung von unterschiedlichen antigenetischen Strukturen, sogenannten "pathogen associated molecular patterns“ (PAMP's) und verschiedener proinflammatorischer Zytokine eine immunologische Reaktion, die letztendlich zur charakteristischen parodontalen Gewebedestruktion führt. Für die kariogene und die parodontopathogene Infektion werden identische Infektionswege diskutiert. Neben der Übertragung durch die Eltern (vertikale Infektion), gilt die Infektion durch Partner und Freunde (horizontale Infektion) als die häufigste Infektionsquelle (Asikainen et al.: JADA 1997; 128: 1263).
Pathophysiologie der Zahnkaries und der marginalen Parodontitis

Hinsichtlich ihrer Pathophysiologie besitzen die dentale Karies und die Parodontitis als bakterielle Infektionserkrankungen teilweise gemeinsame Ursachen. Als zentraler Auslöser für beide Erkrankungen kann die Etab- 
Im Zusammenhang mit der Etablierung von kariösen Defekten gelten aktuell neben der Stärke und Häufigkeit der Fluoridexposition des Zahnhartgewebes vor allem die Qualität der regelmäßigen häuslichen Mundhygiene, die Menge und Pufferkapazität des Speichels sowie die absolute Menge und Frequenz der Ingestion von kariogenen Disacchariden, vor allem von Saccharose, als entscheidende Modulatoren des individuellen Kariesrisikos (Hänsel-Petersson et al.: Caries Res 2002; 36: 327). Wie neuere epidemiologische Studien belegen, ist dabei weniger ein singulärer Faktor alleine als vielmehr das Zusammenspiel aller Faktoren gemeinsam für dieHöhedes individuellen Erkrankungsrisikos determinierend (van Loveren et al. Int Dent J 2002; 52 : 399). Zusätzlich sind einige spezielle Bedingungen bzw.Erkrankungen mit einemerhöhten Kariesrisiko assoziiert. So kann beispielsweise die Eingliederung von festsitzenden kieferorthopädischen Geräten, genetisch bedingte Mineralisationsstörungen der Zahnhartsubstanz (z.B. Amelogenesis imperfecta), eine antidepressive medikamentöse Therapie oder eine onkologische Strahlentherapie im Kopf-Hals-Bereich zu einer massiven Erhöhung des Erkrankungsrisikos beitragen (Folwaczny et al.: Dtsch Zahnärztl Z 2001; 56 : 285, Friedlander et al.: Oral Surg Oral Med Oral Pathol Oral Radiol Endod 2002; 94: 404).

\section{Risikofaktoren für die marginale Parodontitis}

Auf Grund der primären bakteriellen Pathogenese spielt sicher auch für die Parodontitis die Qualität der Mundhygiene eine zentrale Voraussetzung für die Manifestation parodontaler Entzündungen. Zusätzlich ist derzeit der Einfluss von zwei spezifischen Risikofaktoren gesichert. Neben Patienten mit Diabetes mellitus unterliegen auch Individuen mit chronischem Nikotinabusus einem signifikant erhöhten Risiko für eine entzündliche Parodontalerkrankung. Chronischer Nikotinabusus ist in Abhängigkeit von der Intensität mit einem 5- bis 8-fach höheren Erkrankungsrisiko im Vergleich zu Nichtrauchern assoziiert (Johnson et al.: J Periodontol 2004; 75: 196). In der Gruppe der Risikoindikatoren für die marginale Parodontitis werden schließlich der chronische psychosoziale Stress, die Osteoporose sowie verschiedene genetische Prädispositionsfaktoren, zum Beispiel funktionell effektive Polymorphismen im Interleukin $1 \beta-\mathrm{Gen}$, eingeordnet $(\mathrm{Pa}$ ge J: Periodont Res 1999; 34: 331).

Sowohl für die Karies als auch für die Parodontitis scheint für Männer ein höheres Erkrankungsrisiko als für Frauen zu bestehen. Darüber hinaus kommt auch ein Einfluss sozialer Faktoren, insbesondere die Zugehörigkeit zu einer niedrigeren sozialen Schicht, auf das Manifestationsrisiko beider Erkrankungsentitäten in Frage(Salvi et al.: Periodontology 2000 1997; 14: 173).

\section{Präventive Strategien gegen die Zahnkaries und die marginale Parodontitis}

Im Hinblick auf den charakteristischen vertikalen Übertragungsweg der kariogenen und parodontopathogenen Bakterien sollten präventive Strategien idealerweise bereits bei der Infektionsübertragung im Kindesalter ansetzen. Im Sinne der sogenannten Primärprimärprophylaxe kann das Risiko einer erfolgreichen Übertragung der krankheitsauslösenden Mikroorganismen durch die sorgfältige Behandlung der dentalen Karies und der marginalen Parodontitis bei den Eltern deutlich verringert werden (Günay et al.: Clin Oral Invest 1998; 2: 137). Nach aktuellen pathogenetischen Modellen spielt neben der Art der von den Eltern übertragbaren Bakterien vor allem auch das Überschreiten einer

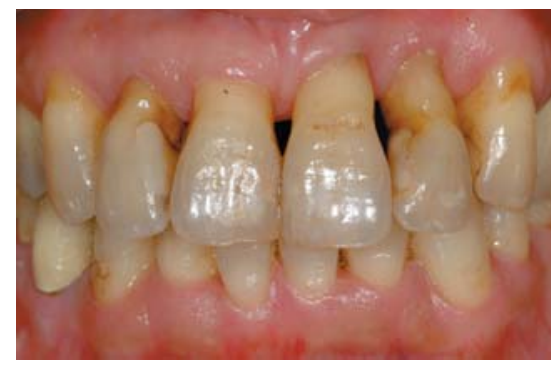

Abb. 2 Chronische Parodontitis: Der Parodontitis-assoziierte Verlust des Alveolarknochens verursacht eine klinisch sichtbare Freilegung der zervikalen Abschnitte der Zahnwurzel (Quelle: M. Folwaczny).

kritischen Infektionsdosis eine entscheidende Rolle für die erfolgreiche vertikale Erkrankungsübertragung.

Die Primärprävention der dentalen Karies und der marginalen Parodontitis zielt im Hinblick auf die im wesentlichen infektiöse Pathogenese in erster Linie auf die Sicherstel-

\section{Präventive Strategien gegen dentale Karies und marginale Parodontitis}

Infektionsprophylaxe durch Behandlung einer floriden Zahnkaries und einer marginalen Parodontitis. Mundhygiene: Störung der Reifungsprozesse der kariogenen und parodontopathogenen Bakterienflora. Diät zur Karies-Prävention: Verringerung der Ingestion von kariogenen Disacchariden.

Fluoridexposition zur Karies-Prävention: verstärkte Remineralisation auf der Oberfläche des Zahnschmelzes, Behinderung des bakteriellen Stoffwechsels (Glykolyse) und des intrazellulären Protonenhaushalts.

Fissurenversiegelung zur Karies-Prävention: Schutz der Zahnoberfläche vor Anlagerung eines mikrobiologischen Biofilms (dentale Plaque), Unterbrechung der Substratzufuhr für etablierte Bakterien.

Zahnärztliche Kontrolle: Frühzeitige Diagnose und Behandlung der Zahnkaries und der marginalen Parodontitis, Kontrolle und Remotivation der häuslichen Mundhygiene (Plaqueindizes), Kontrolle und Einstellung einer möglichst wenig kariogenen Diät.

Blutzuckerkontrolle bei Diabetes mellitus zur Parodontitis-Prävention: Wechselwirkung zwischen Qua lität der Blutzuckerkontrolle und parodontalem Erkrankungsrisiko sowie der Effektivität einer parodontalen Behandlung, zusätzlich bessere Blutzuckerkontrolle bei guter parodontaler Gesundheit.

Nikotinkarenz zur Parodontitis-Prävention: Reduktion des Nikotinabusus verringert das parodontale Erkrankungsrisiko und verbessert die Heilungsprognose.

\section{Präventive Strategien gegen orale Malignome}

Alkohol- und Nikotinkarenz verringern das Erkrankungsrisiko für orale Plattenepithelkarzinome.

Ärztliche bzw. zahnärztliche Kontrolle: Frühzeitige Diagnose und Behandlung maligner Mundschleimhautveränderungen, Diagnose und Kontrolle präkanzeröser Mundschleimhautveränderungen, Sanierung und Erhaltung der dentalen Gesundheit. 

mechanischen Mundhygiene. Neben der Reinigung der Zähne wird durch die übliche Verwendung von fluoridhaltigen Zahnpasten gleichzeitig eine zweite wichtige prophylaktische Säule der Kariesprävention gestärkt. Auf Grund der in Deutschland nicht praktizierten Fluoridierung des Trinkwassers, stellen fluoridhaltige Zahnpasten neben fluoridhaltigem Speisesalz die wichtigste Quelle der dentalen Fluoridprophylaxe dar (Twetman et al.: Acta Odontol Scand 2003; 61: 347).

Die sogenannte Fissurenversiegelung, also die Versiegelung von retentiven Strukturen der Kaufläche von Molaren und Prämolaren mit Hilfe von lackartigen Materialien, dient sowohl der primären als auch der sekundären Prävention (Kühnisch et al:: Dtsch Zahnärztl Z2003; 58: 620). So soll die Fissurenversiegelung nicht nur bislang kariesfreie Bereiche der Kaufläche, die wegen ihrer retentiven Eigenschaften einem erhöhten Kariesrisiko unterliegen, schützen, sondern zusätzlich bereits vorhandene kariöse Initialläsionen am weiteren Fortschreiten hindern (Mejare et al.: Acta Odontol Scand 2003; 61: 321).

Ebenfalls primär- und sekundärpräventive Ziele verfolgt die meistens in halbjährlichen Abständen empfohlene zahnärztliche Untersuchung der Mundhöhle. Neben der frühzeitigen Diagnose und Behandlung von oro-dentalen Erkrankungen kann durch die Beurteilung der Qualität der Mundhygiene mit Hilfe von Plaqueindices sowie die Instruktion und Remotivation zur regelmäßigen Mundhygiene die primäre Vorbeugung der dentalen Karies sowie der Parodontitis gestärkt werden (Salvi et al.: JClin Periodontol 2003; 30: 840). Wegen des hohen Einflusses der Exposition gegenüber kurzkettigen Kohlenhydraten, insbesondere der Saccharose, sollten präventive Strategien immer auch die Einstellung einer möglichst wenig karioge- nen Diät einbeziehen. Neben der Reduktion des absoluten Anteils an zuckerhaltigen Nahrungsmitteln zielt dieser Aspekt der oralen Prävention vor allem auch auf die Verringerung der Frequenz der Ingestion von Kohlenhydraten mit kariogener Potenz. Zur Abschätzung der Intensität der oralen Aufnahme von zuckerhaltigen Nahrungsmitteln kann die Stärke der oralen Infektion durch Lactobacillus spp. mikrobiologisch bestimmt werden (Hänsel Petersson et al.: Caries Res 2002; 36: 327).

den Karzinomen der Speicheldrüsen. Für die Etablierung eines oralen Plattenepithelkarzinoms gelten der chronische Nikotin- und Alkoholabusus als die beiden wichtigsten Risikofaktoren. Zudem ist ein schlechter Gebisszustand als unabhängiger Risikofaktor mit der Entstehung eines Karzinoms der Mundschleimhaut assoziiert (Lissowska et al.: Eur J Cancer 2003; 12: 25). Als Präkanzerosen des Plattenepithelkarzinoms werden neben der Leukoplakie und Erythroplakie vor allem auch der orale Lichen planus diskutiert.

Spezielle präventive Aspekte bei der Parodontitis

Die primäre und sekundäre Prävention der marginalen Parodontitis sollte zusätzlich auf die erfolgreiche Behandlung des chronischen Nikotinabusus zielen. Neben der signifikanten Reduktion des primären Erkrankungsrisikos wirkt sich die Nikotinkarenz zudem auch verbessernd auf die Behandlungsfähigkeit und Prognose einer bereits klinisch etablierten Parodontitis aus. Diese Aussage wird eindrucksvoll durch die Beobachtung belegt, wonach die sogenannte therapierefraktäre Parodontitis in 90\% der Fälle bei Rauchern anzutreffen ist (MacFarlane et al.: J Periodontol 1992; 63: 908). Das erhöhte parodontale Erkrankungsrisiko von Diabetikern kann durch eine optimale Blutzuckerkontrolle deutlich reduziert werden (Mealey et al::JPeriodontol 2000; 71: 664). Erwähnenswert ist in diesemZusammenhang auch die wechselseitige Beeinflussung von Diabetes mellitus und marginaler Parodontitis. Neben einer deutlich vergößerten Prävalenz von entzündlichen Parodontalerkrankungen bei Diabetikern konnte auch ein Zusammenhang zwischen der Qualität der medikamentösen Blutzuckerkontrolle und einer begleitend bestehenden Parodontalerkrankung gezeigt werden (Rodrigues et al.: J Periodontol 2003; 74: 1361).

\section{Prävention von oralen Malignomen}

Unter den Malignomen der Mundhöhle findet sich mit einem Anteil von 90-94\% mit weitem Abstand am häufigsten das Plattenepithelkarzinom (Daley et al.: J Can Dent Assoc 2003; 69: 577). Die restlichen Fälle rekrutieren sich im wesentlichen aus den Sarkomen der unterschiedlichen oro-dentalen Hartund Weichgewebearten, Melanomen sowie
Aus diesen Feststellungen ergeben sich unmittelbar die präventiven Strategien: Neben der Reduktion bzw. dem vollständigen Verzicht auf einen regelmäßigen Alkohol- und Nikotinkonsum ist vor allem die frühzeitige Diagnose und Verlaufskontrolle der präkanzerösen Mundschleimhautveränderungen zur Vorbeugung eines Mundhöhlenkarzinoms anzustreben. Im Hinblick auf die deutlich bessere Heilungsprognose von Plattenepithelkarzinomen in frühen Stadien dient die regelmäßige Kontrolle der Mundschleimhaut und die frühzeitige therapeutische Intervention nicht nur der primären sondern gleichzeitig auch der sekundären Prävention. Nach einer aktuellen Studie scheint neben dem Tumorstadium vor allem die Dicke des Tumors die wesentliche prognostische Variable für die individuelle Heilungschance darzustellen (Sheahan et al.: Otolaryngol Head Neck Surg 2003; 129: 571).

Korrespondenz

Priv.-Doz. Dr. med. Dr. med. dent. Matthias Folwaczny, Poliklinik für Zahnerhaltung und Parodontologie Goethestr. 70 80336 München

DOI: 10.1055/s-2004-831359

Fachliche Betreuung der
„Serie Prävention“:
Prof. Dr. Dr. h. c. Peter C. Scriba
Medizinische Klinik / Klinikum Innenstadt
der Universität München
Ziemssenstr. 1
80336 München
Prof. Dr. Friedrich W. Schwartz
Medizinische Hochschule Hannover
Abteilung für Epidemiologie, Sozialmedizin
und Gesundheitsforschung
Carl-Neuberg-Str. 1
30623 Hannover

Ludwig-Maximilians Universität 